\title{
CLAREAMIENTO DENTAL: GUÍA PARA EL PACIENTE
}

\section{TOOTH BLEACHING: PATIENT GUIDE}

\author{
Cedillo-Orellana Ivanna ${ }^{1}$ \\ 1 Od Esp, Docente de pregrado en Endodoncia Universidad Católica de Cuenca.Ecuador \\ *scedilloo@ucacue.edu.ec
}

\begin{abstract}
Resumen
El presente artículo de opinión pretende informar al odontólogo acerca de una práctica de gran demanda dentro de la odontología estética actual el çlareamiento dental en dientes vitales", explica las causas de coloración dentaria, las diferentes alternativas de tratamiento para lograr una armonía óptica de las piezas dentales, indicaciones, precauciones y contraindicaciones; con la finalidad de que compartan esta información a sus pacientes.
\end{abstract}

Palabras clave: Blanqueamiento en dientes vitales, Peróxido de Hidrógeno, Peróxido de Carbamida.

\begin{abstract}
This opinion's article aim is to inform patients about a practice of great demand within the current aesthetic dentistry "dental bleaching on vital teeth", explains the causes of tooth coloration, different treatment alternatives to achieve an optical harmony of the teeth, indications, precautions and limitations of dental whitening; with the purpose to share this information to their patients.
\end{abstract}

Key words: Vital tooth bleaching, Hydrogen Peroxide, Carbamide Peroxide.

\section{INTRODUCCIÓN}

El clareamiento dental externo mal llamado blanqueamiento dental, es un proceso dinámico en el que mediante la aplicación de productos químicos, por lo general geles, sobre la estructura dental se logra aclarar el tono de los dientes más no pintarlos de blanco, en tal sentido el límite del clareamiento dependerá de la estructura del diente, los pigmentos que se encuentren impregnados en él y el tiempo de permanencia de estos. ${ }^{1,2}$ Este procedimiento se ha vuelto cada vez más popular debido a la gran demanda estética de los últimos años, sin embargo, la preocupación de los odontólogos en cuanto a esta terapia se presentó desde hace mucho tiempo atrás, antiguamente se utilizaron productos como ácido oxálico, cloro e incluso cianuro de potasio, los cuales no aportaban resultados favorables y tenían propiedades tóxicas por lo que están en desuso. Fue en la década de 1884 que Harlan usa por primera vez el Peróxido de Hidrógeno como agente clareador, el cuál hasta la fecha es usado junto con sus subproductos ya que ha demostrado buenos resultados. ${ }^{3,4}$ Actualmente existe una sobreoferta de estos procedimientos tanto en consultorios dentales, como productos de uso doméstico e incluso combinaciones de ambos. Sin embargo, este tratamiento debería ser aplicado después de una evaluación odontológica, ya que no se lo puede realizar en todos los pacientes y debe ser supervisado por un profesional en el área. ${ }^{5}$ El clareamiento dental es un tratamiento útil principalmente en pacientes con pigmentaciones adquiridas por el consumo de alimentos con exceso de colorantes y hábitos como fumar, existen algunas otras condiciones dentales que también requieren de terapias de clareamiento (amelogénesis imperfecta y fluorosis) con resultados que varían según el caso. ${ }^{6}$

Se considera al clareamiento una técnica segura cuando esta es usada adecuadamente y en pacientes que presenten un estado de salud bucal idóneo, pese a esto y debido a la difusión de los agentes clareadores y sus subproductos en la estructura del diente se pueden presentar efectos secundarios no deseados, principalmente sensibilidad dental la cual es generalmente reversible. ${ }^{7,8} \mathrm{El}$ objetivo de este artículo es brindar los conocimientos básicos y recomendaciones que podrá transmitir el odontólogo a sus pacientes con la finalidad de que estos procedimientos se realicen bajo condiciones óptimas y con expectativas reales por parte del paciente.

De igual forma este articulo busca concienciar a los pacientes respecto a los efectos secundarios que se pudieran presentar tras este tratamiento.

Para lograr esta serie de recomendaciones, se ha realizado una búsqueda de información relevante y actualizada, con la finalidad de dar pautas de divulgación científica, que significa traducir el lenguaje técnico a un lenguaje más universal. 


\section{ESTADO DEL ARTE}

\subsection{Causas de coloración dentaria.}

La evidencia científica actual, reporta que las pigmentaciones y oscurecimiento dental pueden ser por dos causas:

-Factores intrínsecos (de causa interna).- Las tinciones de origen intrínseco se presentan debido a factores congénitos y/o adquiridos, pudiendo estar relacionados con fluorosis, amelogénesis o dentinogénesis imperfecta y el uso de tetraciclinas. ${ }^{9,10}$

-Factores extrínsecos (de causa externa).- Las de origen extrínseco se asocian al consumo de alimentos con alto contenido de colorantes ya sean estos naturales o artificiales, a hábitos como fumar, mala higiene e incluso al uso por periodos prolongados de enjugues bucales medicados y con colorantes. ${ }^{10,11}$

\section{2 ¿En qué casos se usan agentes los agentes de clarea- miento dental?}

La necesidad de clareamiento dental responde a fines estéticos y restauradores en los que se requiera eliminar manchas provocadas por factores extrínsecos e incluso pigmentaciones de origen intrínseco siempre y cuando el caso lo amerite y el paciente presente las condiciones bucales adecuadas. El uso de estos productos químicos se puede iniciar únicamente luego de un examen odontológico y cuando el profesional en el área así lo indique. ${ }^{12}$

\subsection{Alternativas del tratamiento}

Existen tres alternativas para este tratamiento: ${ }^{13}$

a) En el consultorio Odontológico: En este caso el odontólogo es el encargado de aplicar el agente clareador y de controlar los resultados. Se trata de varias aplicaciones del producto de alta concentración en una o varias citas; los resultados se pueden observar en corto tiempo.

b) De uso doméstico: El paciente es el responsable de cumplir el tratamiento, así mismo de controlar los resultados. Por lo general es una aplicación diaria de un gel de baja concentración con el cual se obtienen resultados en un período de tiempo mayor que en el caso anterior.

c) Combinado: Cuando el tratamiento de consultorio es complementado con la aplicación doméstica de geles clareadores. Aplica en casos en los que la aplicación de consultorio no es suficiente para cumplir las expectativas del paciente y este no presenta sensibilidad dental por lo que se puede continuar con el uso de estos productos químicos.

La elección de la técnica dependerá del tipo, tiempo, causa e intensidad de la pigmentación, así como las características de las piezas dentales del paciente y la presencia o no de sensibilidad dental. ${ }^{14}$

Los productos químicos más usados para este fin son el Peróxido de Hidrógeno y el Peróxido de Carbamida, siendo generalmente el primero para uso en el consultorio y el segundo para uso doméstico. ${ }^{1}$

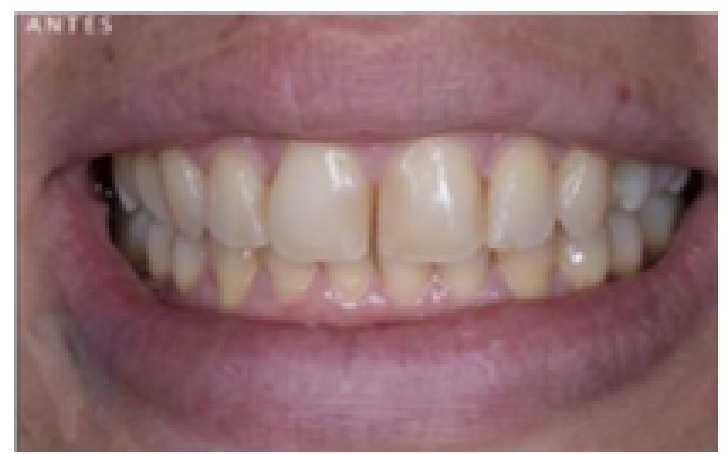

Fig. 1. Fig. 1: Pigmentaciones previas al tratamiento con clareamiento dental en consultorio.

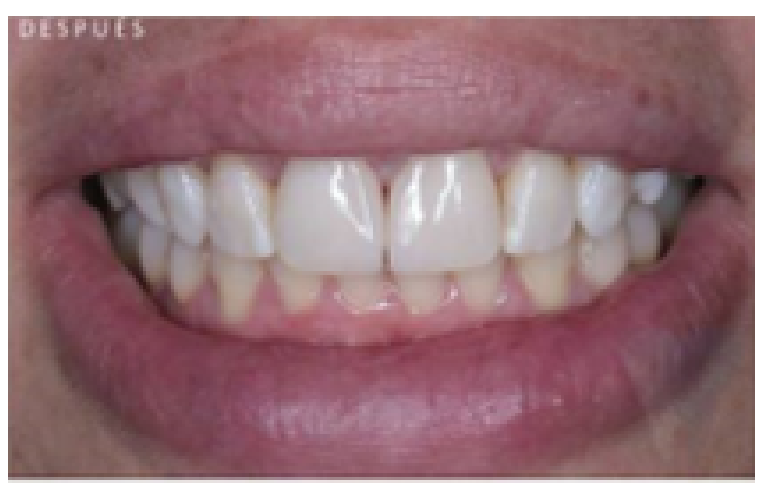

Fig. 2. Fig. 2: Resultado obtenido luego del tratamiento de clareamiento dental en consultorio.

\section{MECANISMO DE ACCIÓN DE LOS AGENTES CLAREADORES}

Todos los agentes clareadores tienen un principio de acción similar, que consiste en la aplicación de un químico en la superficie del diente el cual penetra e inicia una serie de reacciones químicas con los pigmentos (moléculas de gran tamaño) los cuales son fraccionados en moléculas más pequeñas, fáciles de eliminarlas de la superficie dental. ${ }^{13}$ Otra acción que tienen los agentes blanqueadores es la modificación de la estructura de la superficie dental consiguiendo de esta manera que la refracción de la luz cambie, y el diente por un fenómeno óptico se vea más claro. Estos dos mecanismos son los más aceptados por la literatura científica actual. ${ }^{14}$

\subsection{Precauciones y Contraindicaciones.}

Previo al tratamiento es conveniente una evaluación de la salud bucal. La presencia de caries dental, restauraciones 
defectuosas, inflamación gingival, recesiones, fisuras, fracturas y otras características que se consideren importantes, deben ser tratadas antes del procedimiento. El uso de agentes para clareamiento está contraindicado en casos en los que no se pueda solucionar los problemas detallados anteriormente. ${ }^{15,16}$ Otra de las precauciones que se debe considerar es evitar dispositivos que generen calor excesivo como los usados en el famoso "blanqueamiento láser", en el que se usan fuentes de luz halógena o luz led que calientan el producto y permiten que actúen más rápido pero no mejor, este aumento de temperatura puede causar sensibilidad dental severa e incluso daños irreversibles en la vitalidad de la pieza dental. ${ }^{17}$

\subsection{Consecuencias del Clareamiento Dental.}

Estudios describen a la sensibilidad dental como la consecuencia más común, la cual es generalmente reversible y puede ocurrir durante o luego del aclaramiento. En casos en los que el producto es aplicado por tiempo excesivo, a concentraciones elevadas y activado por fuentes que generan calor desmedido se puede generar incluso pérdida de vitalidad de la pieza dental. De ahí la importancia de que el clareamiento sea realizado por un odontólogo capacitado en esta área. Inclusive los clareamientos de uso domiciliario, podrían tener estos efectos secundarios, que son proporcionales al tiempo de aplicación y a la concentración del producto principalmente. ${ }^{18}$

\section{CONCLUSIONES}

El clareamiento dental es un tratamiento que en la actualidad se lo aplica indiscriminadamente debido a la creciente demanda de procedimientos estéticos, sin embargo, no debería ser aplicado en todos los casos y menos aún sin supervisión de un profesional en el área quien luego de un examen bucal exhaustivo con base en fundamento científico y con criterio clínico indicará si es conveniente la aplicación de estas sustancias químicas para mejorar la tonalidad de las piezas dentarias.

La eliminación de los pigmentos de la superficie dental dependerá del tipo de coloración, el tiempo que tenga esta, la intensidad de la mancha y las características de la estructura dental. Por lo que se el paciente deberá saber que no siempre se logrará el tono que desea conseguir sino aquel que se pueda lograr dependiendo de estos factores. Además, se debe tener en cuenta que luego de esta terapia se debe reducir significativamente el consumo de alimentos altos en colorantes para permitir que los resultados se mantengan.

Las modalidades de tratamiento son variadas, la elec- 1 ción de una de estas dependerá de los resultados que se necesiten obtener, el tiempo que se vaya a aplicar y el costo a cubrir; el clareamiento en el consultorio hasta la actualidad es la técnica que brinda mejores resultados en tiempo menor, pero con un costo superior. Los productos más económicos como los de aplicación domiciliaria si bien ayudan a aclarar la tonalidad de las piezas dentales, requieren de varias aplicaciones para evidenciar resultados y al no ser supervisados por un profesional pueden traer consecuencias desagradables.

Finalmente se debe tener presente los potenciales e innegables efectos adversos que podrían ocasionarse, aunque estos sean mínimos. Factores como concentración del producto, tiempo de aplicación y activación del agente clareador pueden influir directamente en la extensión e intensidad del daño, que puede ir desde sensibilidad dental hasta un proceso de necrosis pulpar.

Conflicto de intereses y financiamiento Los autores declaran no tener conflicto de, haber cumplido con los requisitos de autoría y haber autofinanciado este artículo.

\section{Referencias}

1 Zaragoza VM. Bleaching of vital teeth: technique. Estomodeo. 1984; 9:7-30.

2 Soares D, Ribeiro AP, Sacono NT, Coldebella CR, Hebling J, Costa CA. Transenamel and transdentinal cytotoxicity of carbamide peroxide bleaching gels on odontoblast-like MDPC-23 cells. Int End J 2011; 44: 116-125.

3 Kirk EC. The chemical bleaching of teeth. Dent Cosmos 1889; 31: 273-83.

4 Barker G. The cause and treatment of discolored teeth. Dent Cosmos 1861; 3:57-60

5 Aushill T. Efficacity, side effects and patients' acceptance of different bleaching techniques (OTC, in-office, at-home). Oper Dent 2005;30:156-163

6 Goldberg M, Grootveld M, Lynch E. Undesirable and adverse effects of tooth-whitening products: a review. Clin Oral Invest 2010; 14:1-10

7 Edward J.At- home bleaching: pulpal effects and tooth sensitivity issues, part II. Journal Compilation 2006; 18:301305

8 Roderjan D. Response of Human Pulps to Different InOffice Bleaching Techniques: Preliminary Findings. Braz Dent J 2015; 26(3): 242-248

9 Grobler SR, Kotze D. Relationship between wnamel fluoride levels, degree of fluorosis and caries experience in communities with a nearly optimal and a high, fluoride level in drinking water. Caries Res 1986; 20:284-288. 12.

10 10. Gonzalez O. Blanqueamiento dentario: Operatoria Dental. Estética y Adhesión. Ed. Grupo Guía 2003; 22:225. 13. Swift EJ. A method for bleaching discolored teeth. Quintessence 1988; 19:607

1 Goldstein RE, Lancaster J. Survey of patient attitudes toward current esthetic procedures. J Prosthet Dent. 1984; 2:775. Colon P. Improving the appearance for severely fluosed teeth. JADA 1993; 89:1329-1331.

12 Joiner A. The bleaching of teeth: a review of the literature. J Dent 2006;34:412-9. 
13 Kwon S. Review of the Mechanism of Tooth Whitening. J Esthet Restor Dent, 2015; 27: 240-257.

14 Feinman RA, Madray G, Yarborough D. Chemical, optical, and physiologic mechanisms of bleaching products: a review. Pract Periodontics Aesthet Dent 1991;3:32-6.

15 Nathanson D. Vital Tooth Bleaching: Sensitivity and pulpal considerations. J Am Dent Assoc. 1997;128:41s-44

16 Sarrett D. Tooth whitening today. J Am Dent Assoc. 2002;133:1535-1538

17 Michida S, Passos SP, Kimie AR . Intrapulpal temperature variation during bleaching with various activation mechanisms. J Appl Oral Sci 2009;17:436-9.

18 Esposito P, Varvara G, Murmura G, Terlizzi A, Caputi S. Ability of healthy and inflamed human dental pulp to reduce hydrogen peroxide. Eur J Oral Sci 2003;111:454-6.

Recibido: 5 de Enero de 2017

Aceptado: 17 de Marzo de 2017 